Alberta-Thy-18-94

gr-qc/9405033

May 1994

\title{
Quasilocal energy for a Kerr black hole
}

\author{
Erik A. Martinez * \\ Theoretical Physics Institute, \\ Department of Physics, University of Alberta, \\ Edmonton, Alberta T6G 2J1, CANADA.
}

\begin{abstract}
The quasilocal energy associated with a constant stationary time slice of the Kerr spacetime is presented. The calculations are based on a recent proposal [1] in which quasilocal energy is derived from the Hamiltonian of spatially bounded gravitational systems. Three different classes of boundary surfaces for the Kerr slice are considered (constant radius surfaces, round spheres, and the ergosurface). Their embeddings in both the Kerr slice and flat threedimensional space (required as a normalization of the energy) are analyzed. The energy contained within each surface is explicitly calculated in the slow rotation regime and its properties discussed in detail. The energy is a positive, monotonically decreasing function of the boundary surface radius. It approaches the Arnowitt-Deser-Misner (ADM) mass at spatial infinity and reduces to (twice) the irreducible mass at the horizon of the Kerr black hole. The expressions possess the correct static limit and include negative contributions due to gravitational binding. The energy at the ergosurface is compared with the energies at other surfaces. Finally, the difficulties involved in an estimation of the energy in the fast rotation regime are discussed.
\end{abstract}

PACS numbers: 04.20.Cv, 05.30.Ch, 97.60.Lf

Typeset using REVTEX

*electronic address: martinez@phys.ualberta.ca 


\section{INTRODUCTION}

Even at the classical level, general relativity differs from other physical theories in that it accepts several alternative 'definitions' of quasilocal energy. Despite considerable efforts, no definite expression for quasilocal energy has yet appeared. In fact, different proposals provide satisfactory definitions of energy when applied to the appropriate physical situations. In the present paper we study the quasilocal energy recently proposed in Ref. [1] as applied to a Kerr black hole [2].

One of the appealing features of the definition of quasilocal energy adopted in this paper is its straightforward derivation from the gravitational action for a spatially bounded region [1]. Consider a spacetime $\mathcal{M}$ foliated by spacelike hypersurfaces denoted by $\Sigma$. The spacetime is spatially bounded by the three-dimensional surface ${ }^{3} B$, and the intersection of $\Sigma$ with the boundary ${ }^{3} B$ is a two-dimensional surface ${ }^{2} B$ with induced two-metric $\sigma_{a b}$. The quasilocal energy of $\Sigma$ contained within the two-dimensional boundary ${ }^{2} B$ arises from the action as the value of the Hamiltonian that generates unit time translations in ${ }^{3} B$ orthogonal to the surface ${ }^{2} B$. It can be expressed as the proper surface integral [1]

$$
E=\varepsilon-\varepsilon^{0} \equiv \frac{1}{\kappa} \int_{{ }^{2} B} d^{2} x \sqrt{\sigma}\left(k-k^{0}\right)
$$

involving the trace $k$ of extrinsic curvature of ${ }^{2} B$ as embedded in $\Sigma$. The extrinsic curvature is defined so that $k$ equals (minus) the expansion of the unit outward-pointing spacelike normal to ${ }^{2} B$ in $\Sigma$, and units are chosen so that $G=c=\hbar=1$, and $\kappa \equiv 8 \pi$. This expression for the energy also appears in the study of self-gravitating systems in thermal equilibrium, where it plays the role of the thermodynamical energy conjugate to inverse temperature [3, 4 . A definition of quasilocal energy intimately related to the energy (1.1) has been proposed in Ref. [5]. For a discussion of quasilocal energy proposals, see Refs. [1.6] and references cited therein.

The expression (1.1) includes a subtraction term $\varepsilon^{0}$. This term reflects the subtraction term proposed in Ref. [7] for the gravitational action and represents a normalization of the 
energy with respect to a reference space. The reference space is a fixed hypersurface of some fixed spacetime and $k^{0}$ the trace of extrinsic curvature of a two-dimensional surface in this space whose induced metric is $\sigma_{a b}$. The proposal (1.1) is only sensible if the metric $\sigma_{a b}$ can be embedded uniquely in the reference space. The reference space is usually assumed to be a flat three-dimensional slice $E^{3}$ of flat spacetime. In this case several theorems exist [1] that guarantee that the function $k^{0}$ is uniquely determined for all topologically spherical surfaces with positive curvature two-metrics. To specify the quasilocal energy one requires therefore the intrinsic metric of the surface ${ }^{2} B$ and its embedding in $\Sigma$ as well as the embedding in the three-dimensional reference space $E^{3}$ of a surface whose intrinsic geometry equals that of ${ }^{2} B$.

The energy (1.1) has been previously calculated for static spacetimes [1], where the negative contributions to the energy arising from gravitational binding have been studied. It is of interest to extend this analysis to stationary spacetimes, and particularly to Kerr black holes. Besides its possible astrophysical applications, this analysis is necessary in the study of rotating black holes in thermal equilibrium with a heat bath. For instance, one of the boundary data specified at the boundary surface for the density of states in a microcanonical ensemble description of rotating black holes [8, 4 ] is precisely the quasilocal energy (1.1).

We evaluate in what follows the quasilocal energy (1.1) for constant stationary time Kerr slices $\Sigma$ spatially bounded by three different types of boundary surfaces ${ }^{2} B$ with two-sphere topology. However, it is not easy to evaluate (1.1) exactly. Besides the technical problems arising from the complicated structure of the spacetime, it is not possible to embed an arbitrary two-dimensional boundary surface of the Kerr space $\Sigma$ in $E^{3}$ and therefore to calculate the subtraction term $\varepsilon^{0}$. While exact results will be provided whenever possible, most of the energy calculations have to be performed in the slow rotation regime. This regime consists of assuming $|a| / r \ll 1$ (with $a$ denoting the specific angular momentum of the black hole and $r$ an appropriately defined radial distance), but imposes no constraints on the behavior of the ADM mass $M$ [9]. Whilst this approximation cannot give a fair 
description of the fast rotation regime, it is nevertheless physically interesting and allows one to study the effects of angular momentum on the quasilocal energy. We discuss in detail the embedding for all choices of boundary surface in this approximation and estimate separately the terms $\varepsilon$ and $\varepsilon^{0}$.

In section II we present general expressions for the proper integrals involved in the quasilocal energy for arbitrary stationary axisymmetric spacetimes (that is, without assuming the Kerr metric form). These expressions will be useful to calculate not only $\varepsilon$ for different choices of surfaces embedded in the Kerr slice $\Sigma$ but also $\varepsilon^{0}$ for appropriate surfaces embedded in $E^{3}$. In section III we consider the quasilocal energy contained within two-surfaces ${ }^{2} B$ defined by constant value of the Boyer-Lindquist radial coordinate. These surfaces are natural counterparts (in the case of non-zero angular momentum) to surfaces of constant Schwarzschild radius. They are naturally adapted to the coordinates and considerably simplify the calculation of the energy. In particular, the outer horizon of the Kerr spacetime is a surface of this type. We then present various properties and limiting values of the energy for these surfaces and discuss the contributions due to gravitational binding.

We turn in section IV to the energy contained within a round spherical boundary of the Kerr slice $\Sigma$. There are two advantages in the use of round spheres in the present calculation: firstly, a sphere can be always embedded in flat three-dimensional space and consequently the subtraction term $\varepsilon^{0}$ can be easily determined. Secondly, a spherical surface is particularly useful in the study of black hole thermodynamics. Besides the quasilocal energy mentioned above, the boundary data for rotating black holes in a microcanonical ensemble include a chemical potential (associated with the conserved angular momentum) and the two-geometry $\sigma$ of the two-dimensional boundary ${ }^{2} B$ [4, 8 ]. The ADM mass $M$ and specific angular momentum $a$ of rotating black hole configurations in thermal equilibrium inside the cavity are not free parameters but have to be determined as functions of those boundary data by inverting the boundary data equations [3, [4]. Though this procedure will not be discussed here, it is greatly simplified whenever the boundary surface is a sphere; in this case the information about the two-geometry of the boundary surface is fully contained 
in the area of the sphere, which does not depend explicitly on the parameters $M$ and $a$ [4]. The quasilocal energy expressions obtained in this section are then contrasted in the appropriate limits with the corresponding expressions obtained in section III.

The quasilocal energy within the ergosurface is calculated in section V. The result is contrasted with the energy at the horizon evaluated in section III, with the energy within a $r=2 M$ surface, and with the energy within a constant radius surface whose area equals the surface area of the ergosurface. We conclude with a summary of the results and some comments regarding their generalization beyond the slow rotation regime.

\section{GENERAL EXPRESSIONS}

Consider a four-dimensional stationary axisymmetric spacetime. A three-dimensional axisymmetric spacelike hypersurface $\Sigma$ of this spacetime is described by the line element

$$
h_{i j} d x^{i} d x^{j}=b^{2} d y^{2}+c^{2} d \vartheta^{2}+d^{2} d \varphi^{2},
$$

where $x^{i}(i=y, \vartheta, \varphi)$ denote arbitrary coordinates adapted to the symmetry and the metric functions $b, c$, and $d$ depend only on the 'radial' coordinate $y$ and the 'azimuthal' coordinate $\vartheta$. An arbitrary two-dimensional axisymmetric $\operatorname{surface}^{2} B$ (with the topology of a two-sphere) embedded in the three-dimensional space $\Sigma$ is defined by the relation $y=F(\vartheta)$, where $F$ is a function of the azimuthal angle and the parameters of the solution. Its two-dimensional line element is of the form

$$
\sigma_{a b} d x^{a} d x^{b}=\left(b^{2}{F^{\prime}}^{2}+c^{2}\right) d \vartheta^{2}+d^{2} d \varphi^{2},
$$

where a prime denotes differentiation with respect to the coordinate $\vartheta$. The functions $b, c$, and $d$ in (2.2) are evaluated at $y=F(\vartheta)$.

Let $n^{i}$ denote the unit outward-pointing spacelike normal to ${ }^{2} B$ as embedded in the hypersurface $\Sigma$. In terms of metric functions its components are

$$
\left(n^{y}, n^{\vartheta}, n^{\varphi}\right)=\frac{1}{\sqrt{c^{2}+b^{2} F^{\prime 2}}}\left(c / b,-b F^{\prime} / c, 0\right) .
$$


The extrinsic curvature of the two-surface ${ }^{2} B$ as embedded in $\Sigma$ is denoted by $k_{\mu \nu}$. Its trace $k$ represents (minus) the expansion of the normal vector $n^{i}$ and can be written in terms of a Lie derivative with respect to $n^{i}$ as $k=-\mathcal{L}_{n} \ln \sqrt{h}$. Using (2.3) and the notation $\alpha \equiv c^{2} d$, $\beta \equiv b^{2} d F^{\prime}, \lambda \equiv c^{2}+b^{2} F^{\prime 2}$, and $\delta \equiv \ln \lambda$, the trace is

$$
k=-\left.\frac{1}{b c d}\left[\left(\alpha \lambda^{-1 / 2}\right)_{, y}-\left(\beta \lambda^{-1 / 2}\right)_{, \vartheta}\right]\right|_{y=F(\vartheta)},
$$

while its proper surface integral becomes

$$
\frac{1}{\kappa} \int_{2_{B}} k \sqrt{\sigma} d \vartheta d \varphi=-\left.\frac{1}{4} \int_{0}^{\pi} d \vartheta \frac{1}{b c}\left(\alpha_{, y}-\beta_{, \vartheta}-\frac{\alpha}{2} \delta_{, y}+\frac{\beta}{2} \delta_{, \vartheta}\right)\right|_{y=F(\vartheta)} .
$$

This integral is evaluated at the surface $y=F(\vartheta)$. Both integrals in expression (1.1) are of the general form (2.5), but each one involves different values for the functions $b, c$, and $d$ as well as different coordinate definitions.

Consider in particular the Kerr line element expressed in Boyer-Lindquist [10,11] coordinates $(t, r, \theta, \phi)$ in which the rotational Killing vector field takes the form $\xi_{\phi}^{\mu}=(\partial / \partial \phi)^{\mu}$. The spacelike hypersurfaces $\Sigma$ are slices of constant stationary time $t$. Their line element in BoyerLindquist coordinates is of the form (2.1) with $b^{2} \equiv \rho^{2} / \Delta, c^{2} \equiv \rho^{2}$, and $d^{2} \equiv \Sigma^{2} \sin ^{2} \theta / \rho^{2}$. The Kerr functions $\rho, \Delta$, and $\Sigma$ are functions of position and the parameters $(M, a)$ of the solution, and are explicitly given by

$$
\begin{aligned}
\rho^{2} & =r^{2}+a^{2} \cos ^{2} \theta, \\
\Delta & =r^{2}-2 M r+a^{2}, \\
\Sigma^{2} & =\left(r^{2}+a^{2}\right)^{2}-\Delta a^{2} \sin ^{2} \theta .
\end{aligned}
$$

(The metric function $\Sigma^{2}$ is not to be confused with the spacelike hypersurfaces denoted by $\Sigma$.) The symbol $M$ denotes the ADM mass of the Kerr black hole and $a \equiv J / M$ denotes its specific angular momentum. For non-extremal black holes $|a| \leq M$. A two-dimensional surface ${ }^{2} B$ in $\Sigma$ is described by the equation $r=f(\theta ; M, a)$, and its line element is of the form (2.2). It is not difficult to see from the structure (2.6) of the Kerr metric functions that the evaluation of $\varepsilon$ for the Kerr slice $\Sigma$ bounded by the surface $r=f(\theta ; M, a)$ is technically involved. 
For later purposes we note that the line element of ${ }^{2} B$ in the slow rotation approximation becomes

$$
\begin{aligned}
d s^{2} & \approx\left(1+\frac{a^{2}}{r^{2}} \cos ^{2} \theta\right)\left[\left(1-\frac{2 M}{r}+\frac{a^{2}}{r^{2}}\right)^{-1}\left(\frac{d f}{d \theta}\right)^{2}+r^{2}\right] d \theta^{2} \\
& +r^{2}\left[1+\frac{a^{2}}{r^{2}}\left(1+\frac{2 M}{r} \sin ^{2} \theta\right)\right] \sin ^{2} \theta d \phi^{2}
\end{aligned}
$$

where $r=f(\theta ; M, a)$, and terms of order $O\left(1 / r^{4}\right)$ and higher have been neglected.

\section{A CONSTANT RADIUS SURFACE}

We calculate first the energy within the simplest choice of surface ${ }^{2} B$, defined by $r=$ $r_{0}=$ constant. We assume that $r_{0} \geq r_{+}$, where $r_{+}$represents the outer horizon of the Kerr black hole. Using (2.5), the integral $\varepsilon$ for the surface $r=r_{0}$ can be written explicitly as

$$
\begin{aligned}
\varepsilon= & -\frac{r_{0}}{2} \sqrt{1-\frac{2 M}{r_{0}}+\frac{a^{2}}{r_{0}^{2}}} \times \\
& \int_{0}^{\pi} d \theta \sin \theta \frac{1+\frac{a^{2}}{r_{0}^{2}}-\frac{a^{2}}{2 r_{0}{ }^{2}}\left(1-\frac{M}{r_{0}}\right) \sin ^{2} \theta}{\sqrt{1+\frac{a^{2}}{r_{0}{ }^{2}} \cos ^{2} \theta} \sqrt{1+\frac{a^{2}}{r_{0}{ }^{2}}\left(1+\cos ^{2} \theta\right)+\frac{2 M a^{2}}{r_{0}{ }^{3}} \sin ^{2} \theta+\frac{a^{4}}{r_{0}{ }^{4}} \cos ^{2} \theta}} .
\end{aligned}
$$

Unfortunately, this integral cannot be expressed in terms of simple functions. Whilst it is

possible that the inclusion of the subtraction term simplifies the total integral (1.1) for the quasilocal energy, we turn our attention to the slow rotation regime defined by $|a| / r_{0} \ll 1$. In this approximation the integral becomes

$$
\begin{aligned}
\varepsilon= & -\frac{r_{0}}{2} \sqrt{1-\frac{2 M}{r_{0}}+\frac{a^{2}}{r_{0}^{2}}} \times \\
& \int_{0}^{\pi} d \theta \sin \theta\left[1-\frac{a^{2}}{2 r_{0}^{2}}\left(\frac{M}{r_{0}}+\cos ^{2} \theta\left(1-\frac{M}{r_{0}}\right)\right)+O\left(\frac{a^{4}}{r_{0}^{4}}\right)\right],
\end{aligned}
$$

where only leading order terms in the expansion around the small parameter $|a| / r_{0}$ are considered. This is easily integrated in terms of polynomials with the result

$$
\varepsilon=-r_{0} \sqrt{1-\frac{2 M}{r_{0}}+\frac{a^{2}}{r_{0}^{2}}}\left[1-\frac{a^{2}}{6 r_{0}^{2}}\left(1+\frac{2 M}{r_{0}}\right)+O\left(\frac{a^{4}}{r_{0}^{4}}\right)\right] .
$$

It is important to note that no approximations have been made inside the square-root appearing in $\varepsilon$ (in fact, the square-root times $r_{0}$ is equal to $\Delta^{1 / 2}$ ). For large values of $r_{0}$, 
$\varepsilon \rightarrow M-r_{0}$. The presence of the divergent term indicates the need of a subtraction term for the energy, which we consider below.

In order to calculate the subtraction term $\varepsilon^{0}$ it is necessary to find a two-dimensional surface immersed in flat three-dimensional space $E^{3}$ whose intrinsic geometry equals the intrinsic geometry of the $r=r_{0}$ surface in the Kerr space. Once this surface is known, the trace $k^{0}$ of its extrinsic curvature as embedded in $E^{3}$ will allow us to calculate $\varepsilon^{0}$. Since the intrinsic geometry of a two-dimensional surface is completely characterized by its scalar curvature, we can obtain the equation for the surface by requiring its scalar curvature to be equal to the scalar curvature of the $r=r_{0}$ surface in the Kerr space. Due to the complicated structure of the Kerr spacetime, the construction will be confined to the regime $|a| / r_{0} \ll 1$.

Using (2.7), the intrinsic line element of the $r=r_{0}$ surface in the Kerr space is

$$
d s^{2}=r_{0}^{2}\left(1+\frac{a^{2}}{r_{0}^{2}} \cos ^{2} \theta\right) d \theta^{2}+r_{0}^{2} \sin ^{2} \theta\left(1+\frac{a^{2}}{r_{0}^{2}}+\frac{2 M a^{2}}{r_{0}^{3}} \sin ^{2} \theta+O\left(a^{4} / r_{0}^{4}\right)\right) d \phi^{2}
$$

and its scalar curvature is readily calculated to be

$$
\Re=\frac{2}{r_{0}^{2}}\left[1+\frac{a^{2}}{r_{0}^{2}}\left(-2 \cos ^{2} \theta+\frac{2 M}{r_{0}}\left(1-4 \cos ^{2} \theta\right)\right)+O\left(a^{4} / r_{0}^{4}\right)\right] .
$$

In our approximation regime the curvature (3.5) is positive for all values of the azimuthal angle $\theta$ and the surface can be embedded globally in three-dimensional Euclidean space $E^{3}$. (It is well known [12] that the Kerr horizon cannot be embedded globally in $E^{3}$ whenever $a^{2}>r_{+}{ }^{2} / 3$, or equivalently, $a>\sqrt{3} M / 2$. In this case part of the surface, centered around the equator, remains embedded in $E^{3}$ while another part, centered around the polar caps, becomes embedded in flat pseudo-Euclidean $\left(P E^{3}\right)$ space.)

Consider now the line element

$$
\begin{aligned}
d s_{\text {flat }}^{2} & =-\left(d x^{0}\right)^{2}+d x^{2}+d y^{2}+d z^{2} \\
& =-\left(d x^{0}\right)^{2}+d \mathcal{R}^{2}+\mathcal{R}^{2} d \Theta^{2}+\mathcal{R}^{2} \sin ^{2} \Theta d \Phi^{2}
\end{aligned}
$$

of a four-dimensional flat spacetime. The flat coordinates $(\mathcal{R}, \Theta, \Phi)$ should not be confused with the Boyer-Lindquist coordinates $(r, \theta, \phi)$. The relationship between both coordinate 
systems is important in the construction of the desired two-dimensional surfaces embedded in flat space and deserves to be discussed. The line element (3.6) can be written in terms of Boyer-Lindquist coordinates by defining

$$
\begin{aligned}
x^{0} & =\int d u+d r \\
x & =(r \cos \tilde{\phi}+a \sin \tilde{\phi}) \sin \theta \\
y & =(r \sin \tilde{\phi}-a \cos \tilde{\phi}) \sin \theta \\
z & =r \cos \theta,
\end{aligned}
$$

where the Kerr-Schild [10,11] coordinates $(u, \tilde{\phi})$ are given by $d u=d t-\left(r^{2}+a^{2}\right) / \Delta d r$, and $d \tilde{\phi}=d \phi-a / \Delta d r$. (In fact, in terms of these coordinates the Kerr line element can be written as the direct sum of two line elements, one of them being the flat element (3.6)). The relationship between $(\mathcal{R}, \Theta, \Phi)$ and $(r, \theta, \phi)$ can be obtained easily by noting that the coordinates $(x, y, z)$ satisfy 10

$$
\frac{x^{2}+y^{2}}{r^{2}+a^{2}}+\frac{z^{2}}{r^{2}}=1
$$

and $x^{2}+y^{2}+z^{2}=\mathcal{R}^{2}$. In particular, one obtains $\mathcal{R}^{2}=r^{2}+a^{2} \sin ^{2} \theta$ and

$$
\cos ^{2} \theta=\frac{r^{2}+a^{2}}{r^{2}+a^{2} \cos ^{2} \Theta} \cos ^{2} \Theta, \sin ^{2} \theta=\frac{r^{2}}{r^{2}+a^{2} \cos ^{2} \Theta} \sin ^{2} \Theta
$$

which in the approximation $|a| \ll r$ reduce to

$$
\begin{aligned}
& \cos ^{2} \theta \approx\left(1+a^{2} / r^{2} \sin ^{2} \Theta\right) \cos ^{2} \Theta \\
& \sin ^{2} \theta \approx\left(1-a^{2} / r^{2} \cos ^{2} \Theta\right) \sin ^{2} \Theta .
\end{aligned}
$$

A flat three-dimensional slice of the flat spacetime (3.6) has line element

$$
d s^{2}=d \mathcal{R}^{2}+\mathcal{R}^{2} d \Theta^{2}+\mathcal{R}^{2} \sin ^{2} \Theta d \Phi^{2}
$$

The equation for the desired two-dimensional surface in the flat slice is denoted by $\mathcal{R}=g(\Theta)$, where $g$ is a function of the azimuthal angle $\Theta$ and the parameters $\left(M, a, r_{0}\right)$ of the surface 
in $\Sigma$. Its intrinsic metric is obtained from (3.11). By virtue of (3.10), it is enough in the slow rotation approximation to assume

$$
g(\Theta)=r_{0}\left(1+\frac{a^{2}}{r_{0}^{2}} \omega(\Theta)+O\left(a^{4} / r_{0}^{4}\right)\right)
$$

where $\omega(\Theta)$, a function of order one, is to be determined. The scalar curvature of this surface is

$$
\Re^{0}=\frac{2}{r_{0}^{2}}\left[1-\frac{a^{2}}{r_{0}^{2}}\left(\omega^{\prime \prime}+\omega^{\prime} \cot \Theta+2 \omega\right)+O\left(a^{4} / r_{0}^{4}\right)\right]
$$

where a prime denotes differentiation with respect to $\Theta$. By equating the scalar curvatures (3.5) and (3.13) and using (3.10) we obtain a differential equation for $\omega(\Theta)$, whose solution is $\omega(\Theta)=-M / r_{0} \cos 2 \Theta+1 / 2 \sin ^{2} \Theta$. This implies that the radius of the surface in $E^{3}$, as a function of the azimuthal angle is given by

$$
\mathcal{R}=g(\Theta)=r_{0}\left[1+\frac{a^{2}}{r_{0}^{2}}\left(\frac{1}{2} \sin ^{2} \Theta-\frac{M}{r_{0}} \cos 2 \Theta\right)+O\left(a^{4} / r_{0}^{4}\right)\right]
$$

This equation describes a surface in flat Euclidean space $E^{3}$ whose intrinsic curvature equals the intrinsic curvature of a $r=r_{0}$ surface in the Kerr slice. This surface, as embedded in $E^{3}$, is plotted in Figure 1 for particular values of $M$ and $a$ that satisfy the condition $a^{2} \ll r_{0}^{2}$. It is a distorted sphere with its major axis along the equator. For fixed $a^{2}$, the surface becomes more oblate as $M$ increases. Its intrinsic metric is given by

$$
d s^{2} \approx r_{0}^{2}\left[1+\frac{a^{2}}{r_{0}^{2}}\left(\sin ^{2} \Theta-\frac{2 M}{r_{0}} \cos 2 \Theta\right)\right]\left(d \Theta^{2}+\sin ^{2} \Theta d \Phi^{2}\right)
$$

whereas its area is

$$
A=4 \pi r_{0}^{2}\left[1+\frac{2 a^{2}}{3 r_{0}^{2}}\left(1+\frac{M}{r_{0}}\right)+O\left(a^{4} / r_{0}^{4}\right)\right] .
$$

The extrinsic curvature $k^{0}$ of the surface (3.14) as embedded in the flat space (3.11), and its proper integral can be calculated now using the expressions (2.4) and (2.5) adapted to the coordinates $(\mathcal{R}, \Theta, \Phi)$. A long but direct computation gives the desired integral

$$
\varepsilon^{0}=\frac{1}{\kappa} \int_{2_{B}} k^{0} \sqrt{\sigma} d \Theta d \Phi=-r_{0}\left[1+\frac{a^{2}}{3 r_{0}^{2}}\left(1+\frac{M}{r_{0}}\right)+O\left(a^{4} / r_{0}^{4}\right)\right] \text {. }
$$


The energy is obtained by subtracting (3.17) from (3.3), with the result

$$
\begin{aligned}
E= & r_{0}\left[1-\sqrt{1-\frac{2 M}{r_{0}}+\frac{a^{2}}{r_{0}^{2}}}\right]+\frac{a^{2}}{6 r_{0}}\left[2+\frac{2 M}{r_{0}}+\left(1+\frac{2 M}{r_{0}}\right) \sqrt{1-\frac{2 M}{r_{0}}+\frac{a^{2}}{r_{0}^{2}}}\right] \\
& +r_{0} O\left(a^{4} / r_{0}^{4}\right),
\end{aligned}
$$

where terms of order $r_{0} O\left(a^{4} / r_{0}^{4}\right)$ and higher are considered negligible. Expression (3.18) is the quasilocal gravitational energy of a (slowly rotating) Kerr slice $\Sigma$ spatially bounded by a surface of constant Boyer-Lindquist radial coordinate $r_{0}$. We stress that no restrictions on the mass $M$ have been imposed in the calculation of the energy expression (3.18).

Several features of the above expression are noteworthy. In the asymptotic limit $r_{0} \rightarrow \infty$, the energy approaches the ADM energy $M$. In the limit of zero angular momentum the quasilocal energy reduces to

$$
E=r_{0}-r_{0} \sqrt{1-\frac{2 M}{r_{0}}},
$$

which, as expected, is the quasilocal energy (within a surface of radius $r=r_{0}$ ) for a Schwarzschild black hole of ADM mass $M$. The small mass limit (or equivalently, the large radius limit) of the energy (3.18) is obtained by assuming $M \ll r_{0}$ in (3.18). In this case

$$
E=M+\frac{M^{2}}{2 r_{0}}+\frac{M^{3}}{2 r_{0}{ }^{2}}+\frac{5 M^{4}}{8 r_{0}{ }^{3}}-\frac{7 M^{2} a^{2}}{6 r_{0}{ }^{3}}+r_{0} O\left(a^{4} / r_{0}{ }^{4}\right),
$$

where terms of order $O\left(M^{4} / r_{0}{ }^{4}\right)$ and $O\left(M^{2} a^{2} / r_{0}{ }^{4}\right)$ have been preserved while terms of order $O\left(a^{4} / r_{0}{ }^{4}\right)$ and higher have been neglected. The first term in (3.20) represents the total energy at spatial infinity while the second term represents (minus) the Newtonian gravitational potential energy associated with building a shell of radius $r_{0}$ and mass $M$ by bringing the individual constituent particles from spatial infinity. As discussed in Ref. [1] for the static case, the energy $E$ in this approximation has the natural interpretation of the total energy associated with assembling the gravitational system starting from the boundary of radius $r_{0}$, and it reflects indeed the energy within this boundary. Notice that no terms of order $r_{0} O\left(a^{2} / r_{0}^{2}\right)$ are present in (3.20). 
It is of interest to invert the energy equation (3.18) to obtain the ADM mass $M$ as a function of the energy $E$, the size $r_{0}$ of the cavity, and the specific angular momentum $a$. This not only illustrates the interpretation of the different terms in the energy expression but also, as mentioned in the introductory paragraphs, has relevance in the study of black hole stable configurations in a gravitational microcanonical ensemble. In the limit of small energy, the desired equation is

$$
M=E\left(1-\frac{E}{2 r_{0}}\right)+O\left(a^{2} E^{2} / r_{0}^{3}\right)
$$

In the limit $a \rightarrow 0$ this expression is exact for all values of $E$ and reduces to the expression for the Schwarzschild ADM mass in terms of boundary radius and energy [3].

Expression (3.18) can be used to calculate the energy at the horizon whenever $|a| \ll r_{+}$ (or equivalently, whenever $|a| \ll M)$. The two-dimensional outer horizon [11] of a Kerr black hole is a topological (but not a round) sphere of constant coordinate radius $r_{0}=r_{+}=$ $M+\left(M^{2}-a^{2}\right)^{1 / 2}$. In the present approximation, $r_{+}=2 M\left(1-a^{2} / 4 M^{2}+O\left(a^{4} / M^{4}\right)\right)$. Since $\varepsilon\left(r_{+}\right)=0$, the energy becomes

$$
\begin{aligned}
E\left(r_{0}=r_{+}\right) & =r_{+}\left[1+\frac{a^{2}}{2 r_{+}{ }^{2}}+O\left(a^{4} / r_{+}{ }^{4}\right)\right] \\
& =2 M\left[1-\frac{a^{2}}{8 M^{2}}+O\left(a^{4} / r_{+}{ }^{4}\right)\right] .
\end{aligned}
$$

Recall now that the irreducible mass $M_{i}$ of a Kerr black hole [13 is proportional to the square root of the surface area of the horizon and can be written as

$$
M_{i}=\frac{1}{2}\left(r_{+}^{2}+a^{2}\right)^{1 / 2}=\frac{r_{+}}{2}\left[1+\frac{a^{2}}{2 r_{+}{ }^{2}}+O\left(a^{4} / r_{+}{ }^{4}\right)\right] \text {. }
$$

Therefore the quasilocal energy (3.22) at the horizon equals twice the irreducible mass,

$$
E\left(r_{0}=r_{+}\right)=2 M_{i}\left[1+O\left(a^{4} / M_{i}^{4}\right)\right]
$$

to leading order in the slow rotation regime. This is a very attractive property of the energy (3.18), valid perhaps beyond the present approximations, and constitutes one of the main results of this paper. 
The quasilocal energy (3.18) contained within the constant radius surface is positive for all values of $r_{0} \geq r_{+}$. This can be seen directly from (3.18), since the two terms of this expression are positive whenever $r_{0}$ is larger than the radius of the outer Kerr horizon. The behavior of the quasilocal energy as a function of $r_{0}$ is illustrated in Figure 2 for $|a| \ll r_{+}$. Notice that the energy monotonically increases from $E\left(r_{0}=\infty\right)=M$ to $E\left(r_{0}=r_{+}\right)=2 M_{i}$. As shown in (3.18), the quasilocal energy has contributions related to the irreducible mass and to rotational energy. The interaction of the different energy contributions is non-linear and shows itself even in the slow rotation approximation.

For later purposes we observe that the energy within a surface of constant radius $r_{0}=2 M$ is

$$
E\left(r_{0}=2 M\right)=2 M\left(1-\frac{|a|}{2 M}+\frac{a^{2}}{8 M^{2}}+O\left(a^{3} / M^{3}\right)\right) .
$$

Notice that $E\left(r_{0}=r_{+}\right)>E\left(r_{0}=2 M\right)$.

Finally, the quasilocal energy for an extreme Kerr hole for which $\left.M=|a| \ll r_{0}\right)$ is

$$
E=M+\frac{M^{2}}{2 r_{0}}+\frac{M^{3}}{2 r_{0}^{2}}+r_{0} O\left(M^{4} / r_{0}^{4}\right)
$$

in agreement with the small mass limit (3.20).

\section{A SPHERICAL TWO-DIMENSIONAL BOUNDARY}

We turn now our attention to the quasilocal energy of the Kerr space $\Sigma$ contained within a round spherical surface ${ }^{2} B=S^{2}$ of curvature radius $R$ and surface area $A=4 \pi R^{2}$. The construction of a round sphere in $\Sigma$ and the energy calculation will be computed in the slow rotation regime $a^{2} \ll R^{2}$ supplemented with the additional condition $M \ll R$. Under this approximation terms of order $O\left(M^{2} a^{2} / R^{4}\right)$ and $O\left(M^{4} / R^{4}\right)$ will be considered while terms of order $O\left(a^{4} / R^{4}\right)$ and higher will be neglected.

The equation $r=h(\theta)$ describing $S^{2}$ in $\Sigma$ can be obtained by requiring its scalar curvature to be 


$$
\Re=\frac{2}{R^{2}},
$$

where $R$ is a positive constant. The function $h$ depends on the azimuthal angle and the parameters $(M, a, R)$, and in the slow rotation approximation takes the form

$$
h(\theta)=R\left(1+\frac{a^{2}}{R^{2}} \eta(\theta)+O\left(a^{4} / R^{4}\right)\right)
$$

where $\eta(\theta)$ is a function of order one. Substitution of (4.2) in (2.7) gives us a surface line element whose intrinsic curvature is

$$
\Re=\frac{2}{R^{2}}\left[1-\frac{a^{2}}{R^{2}}\left(\eta^{\prime \prime}+\eta^{\prime} \cot \theta+2 \eta+2 \cos ^{2} \theta+\frac{2 M}{R}\left(4 \cos ^{2} \theta-1\right)\right)+O\left(a^{4} / R^{4}\right)\right],
$$

where a prime in this section denotes differentiation with respect to the Boyer-Lindquist coordinate $\theta$. By equating the scalar curvatures (4.3) with the scalar curvature (4.1) of a round sphere one obtains a differential equation for $\eta(\theta)$, whose solution is $\eta(\theta)=\frac{M}{R} \cos 2 \theta-$ $\frac{1}{2} \sin ^{2} \theta$. Therefore, a two-dimensional round sphere $S^{2}$ of curvature radius $R$ in a slowly rotating Kerr space $\Sigma$ is described by the equation

$$
r=h(\theta)=R\left[1-\frac{a^{2}}{2 R^{2}} \sin ^{2} \theta+\frac{a^{2} M}{R^{3}} \cos 2 \theta+O\left(a^{4} / R^{4}\right)\right] .
$$

As expected from the behavior of the Boyer-Lindquist coordinates, the coordinate radius of the sphere $S^{2}$ is larger at the poles than at the equator. Its line element in the current approximation is

$$
\begin{aligned}
d s^{2} & =R^{2}\left[1+\frac{a^{2}}{R^{2}}\left(1+\frac{2 M}{R}\right) \cos 2 \theta+O\left(a^{4} / R^{4}\right)\right] d \theta^{2} \\
& +R^{2} \sin ^{2} \theta\left[1+\frac{a^{2}}{R^{2}}\left(1+\frac{2 M}{R}\right) \cos ^{2} \theta+O\left(a^{4} / R^{4}\right)\right] d \phi^{2}
\end{aligned}
$$

It can easily be verified that the area of this surface is $A=4 \pi R^{2}$.

The quasilocal energy integral $\varepsilon$ can now be calculated directly from the general expression (2.5) with $b^{2}=\rho^{2} / \Delta, c^{2}=\rho^{2}$, and $d^{2}=\Sigma^{2} \sin ^{2} \theta / \rho^{2}$, and by replacing the coordinates $(y, \vartheta, \varphi)$ by $(r, \theta, \phi)$. The Kerr metric functions $\rho^{2}, \Delta$, and $\Sigma^{2}$, evaluated at the spherical surface (4.4), are 


$$
\begin{aligned}
& \rho^{2}(\theta)=R^{2}\left[1+\frac{a^{2}}{R^{2}}\left(2 \cos ^{2} \theta-1\right)+\frac{2 a^{2} M}{R^{3}}\left(2 \cos ^{2} \theta-1\right)+O\left(a^{4} / R^{4}\right)\right], \\
& \Delta(\theta)=R^{2}\left[1-\frac{2 M}{R}+\frac{a^{2}}{R^{2}} \cos ^{2} \theta+\frac{a^{2} M}{R^{3}}\left(3 \cos ^{2} \theta-1\right)+\frac{2 a^{2} M^{2}}{R^{4}}\left(1-2 \cos ^{2} \theta\right)+O\left(a^{4} / R^{4}\right)\right], \\
& \Sigma^{2}(\theta)=R^{4}\left[1+\frac{a^{2}}{R^{2}}\left(3 \cos ^{2} \theta-1\right)+\frac{2 a^{2} M}{R^{3}}\left(3 \cos ^{2} \theta-1\right)+O\left(a^{4} / R^{4}\right)\right],
\end{aligned}
$$

in the present approximation. The integrand becomes a function of $(M, a, R)$ and the coordinate $\theta$. A long but direct calculation gives the total integral

$$
\varepsilon=-R\left(1-\frac{2 M}{R}\right)^{1 / 2}\left[1+\frac{M^{2} a^{2}}{R^{4}}\left(1-\frac{2 M}{R}\right)^{-1}+O\left(a^{4} / R^{4}\right)\right] .
$$

As expected, this expression is divergent for large $R$. The subtraction term is easily calculated in the present case since a two-dimensional sphere can always be embedded in $E^{3}$. A straightforward calculation gives $k^{0}=-2 / R$ and

$$
\varepsilon^{0}=-R
$$

By subtracting (4.8) from (4.7) one obtains the total energy, which can be written in our approximation as

$$
E=M+\frac{M^{2}}{2 R}+\frac{M^{3}}{2 R^{2}}+\frac{5 M^{4}}{8 R^{3}}-\frac{M^{2} a^{2}}{R^{3}}+R O\left(a^{4} / R^{4}\right)
$$

This expression gives the quasilocal gravitational energy of a Kerr space $\Sigma$ (with specific angular momentum $a^{2} \ll R^{2}$ and mass $M \ll R$ ) spatially bounded by a round sphere of surface area $A=4 \pi R^{2}$. Observe that the specific angular momentum $a$ shows itself only at fourth order in $E$.

We discuss now some properties of the expression (4.9). The energy tends to $M$ as $R$ tends to infinity and in the zero angular momentum limit one recovers the energy expression (3.19) for a Schwarzschild black hole (this limit is expected since a two-surface of constant radius $r_{0}$ in the Schwarzschild spacetime is also a round sphere.) Observe from expression (4.7) that a round spherical surface of area $A=4 \pi R^{2}$ surrounding a (slowly) rotating Kerr black hole of mass $M \ll R$ contains less energy than a round spherical surface of the same 
area surrounding a Schwarzschild black hole of mass $M$. Figure 3 illustrates the behavior of the energy (4.9) as a function of the quantity $R$ whenever $M \ll R$.

It is interesting to contrast the values of quasilocal energy of $\Sigma$ within different surfaces. Comparing (4.9) with (3.20), we observe that the energy contained within a round sphere of curvature radius $R=l_{0}$ is larger than the energy contained within a topologically spherical surface of Boyer-Lindquist radius $r_{0}=l_{0}$ whenever $l_{0}$ is large (that is, $|a| \ll l_{0}, M \ll l_{0}$ ). We can compare also the energy associated with a round sphere of area $A=4 \pi R^{2}$ with the energy associated with a constant radius boundary of the same surface area. Under our approximation the two areas coincide if

$$
R \approx r_{0}\left(1+\frac{a^{2}}{3 r_{0}^{2}}\left(1+\frac{M}{r_{0}}\right)\right) .
$$

As can be seen by substituting this in (4.9) and comparing the resulting energy with (3.20), the energy within the sphere equals the energy within the constant radius surface in the present approximation whenever $R$ and $r_{0}$ are large. This is not surprising since the relative distortion between the constant radius surface and the sphere is very small for large radii.

\section{ERGOSURFACE}

The timelike limit surface (or ergosurface) of a Kerr black hole is the boundary of the region in which particles travelling on a timelike curve can remain on an orbit of the Killing vector field $\xi_{t}^{\mu}=(\partial / \partial t)^{\mu}$ (and so remain at rest with respect to spatial infinity) [11]. The ergosurface is a topologically spherical surface described by $r=r_{*} \equiv M+\left(M^{2}-a^{2} \cos ^{2} \theta\right)^{1 / 2}$; $r_{*}$ coincides with the outer horizon radius $r_{+}$at the poles and equals $2 M$ at the equator. Since the ergosurface is neither a constant radius surface nor a round sphere, expressions

(3.18) and (4.9) cannot be used to estimate the quasilocal energy within it. However, the energy can be evaluated in the slow rotation approximation $|a| \ll M$. The term $\varepsilon$ can in fact be evaluated directly from (2.5) for arbitrary values of angular momentum. The result will not be presented here because, as for previous surfaces, the integral cannot be written 
in terms of simple functions. However, in the small rotation regime the integral can be evaluated explicitly, with the result

$$
\varepsilon=-\frac{\pi|a|}{4}\left(1-\frac{9 a^{2}}{64 M^{2}}+O\left(a^{4} / M^{4}\right)\right)
$$

In order to calculate $\varepsilon^{0}$ we need to construct a surface $\mathcal{R}=l(\Theta ; M, a)$ embedded in $E^{3}$ possessing the same intrinsic geometry as the ergosurface. Since in the slow rotation approximation $r_{*} \approx 2 M\left(1-a^{2} / 4 M^{2} \cos ^{2} \theta\right)$, the intrinsic metric of the ergosurface is, to leading order

$$
d s^{2}=4 M^{2}\left[1+\frac{3 a^{2}}{4 M^{2}} \cos ^{2} \theta\right] d \theta^{2}+4 M^{2}\left[1+\frac{a^{2}}{2 M^{2}}\left(1-\frac{3}{2} \cos ^{2} \theta\right)\right] \sin ^{2} \theta d \phi^{2},
$$

while its scalar curvature is

$$
\Re=\frac{1}{2 M^{2}}\left(1+\frac{3 a^{2}}{4 M^{2}}\left(1-6 \cos ^{2} \theta\right)+O\left(a^{4} / M^{4}\right)\right) .
$$

By equating (5.3) with the scalar curvature of a surface in flat space, we can obtain the desired surface $\mathcal{R}=l(\Theta ; M, a)$ in the regime $|a| \ll M$. A direct calculation gives

$$
l(\Theta ; M, a)=2 M\left[1+\frac{3 a^{2}}{4 M^{2}}\left(1-\frac{3}{2} \cos ^{2} \Theta\right)+O\left(a^{4} / M^{4}\right)\right] .
$$

Notice that the coordinate radius of this surface in $E^{3}$ is larger than $2 M$ at the equator and smaller than $2 M\left(1-a^{2} / 4 M^{2}\right)$ at the poles. The line element of this surface is

$$
d s^{2}=4 M^{2}\left[1+\frac{3 a^{2}}{2 M^{2}}\left(1-\frac{3}{2} \cos ^{2} \Theta\right)+O\left(a^{4} / M^{4}\right)\right]\left(d \Theta^{2}+\sin ^{2} \Theta d \Phi^{2}\right),
$$

while its area is

$$
A=16 \pi M^{2}\left(1+3 a^{2} / 4 M^{2}+O\left(a^{4} / M^{4}\right)\right) .
$$

The extrinsic curvature $k^{0}$ of the surface (5.4) as embedded in flat space provides the subtraction term

$$
\varepsilon^{0}=-2 M\left(1+\frac{3 a^{2}}{8 M^{2}}+O\left(a^{4} / M^{4}\right)\right)
$$


The energy can be obtained now by subtracting (5.7) from (5.1), with the result

$$
E=\varepsilon-\varepsilon^{0}=2 M-\frac{\pi|a|}{4}+\frac{3 a^{2}}{4 M}+\frac{9 \pi|a|^{3}}{256 M^{2}}+M O\left(a^{4} / M^{4}\right) .
$$

This is the quasilocal gravitational energy (in the approximation $|a| \ll M$ ) of a Kerr slice $\Sigma$ spatially bounded by the ergosurface $r=r_{*}$. The above expression shows that the quasilocal energy contained within the ergosurface is positive in the present approximation.

It is interesting to calculate the difference between the energy (3.22) evaluated at the horizon and the energy (5.8) evaluated at the ergosurface. Due to gravitational binding energy contributions, the former is larger than the latter, and

$$
E\left(r=r_{+}\right)-E\left(r=r_{*}\right)=\frac{\pi|a|}{4}-\frac{a^{2}}{M}-\frac{9 \pi|a|^{3}}{256 M^{2}}+M O\left(a^{4} / M^{4}\right) .
$$

This quantity measures the difference between (twice) the irreducible mass and the energy contained within the ergosurface. It is naturally zero when $a=0$.

The surface of coordinate radius $r=2 M$ and the ergosurface $r=r_{*}$ touch at the equator but are well-separated elsewhere, with the former surface enclosing the latter. The energy within the ergosurface is larger than the energy (3.25) within the surface $r=2 M$, and

$$
E\left(r=r_{*}\right)-E(r=2 M)=|a|(1-\pi / 4)+a^{2} / 2 M+M O\left(a^{3} / M^{3}\right) .
$$

To conclude, observe that the area of the constant radius surface $r_{0}=r_{c} \equiv 2 M(1+$ $a^{2} / 4 M^{2}$ ) equals the area of the ergosurface in the approximation $|a| \ll M$. (The former surface is located outside the horizon since, in this approximation, $r_{+} \approx 2 M\left(1-a^{2} / 4 M^{2}\right)$.) From (3.18) the quasilocal energy at this surface is

$$
E\left(r_{c}\right)=2 M\left(1-\frac{|a|}{\sqrt{2} M}+\frac{3 a^{2}}{8 M^{2}}+O\left(a^{3} / M^{3}\right)\right) .
$$

A comparison with (5.8) shows that the energy within the ergosurface is larger than the energy (5.11) contained within the $r=r_{c}$ surface. 


\section{SUMMARY}

We have calculated the quasilocal energy (1.1) for the constant stationary time slice of the Kerr spacetime contained within three different classes of boundary surfaces, namely, constant radius surfaces, round spheres, and the ergosurface. These surfaces were chosen because they play a special role in the physics of Kerr black holes. The calculations were confined to the slow rotation regime $|a| \ll r$. Unless explicitly stated, the mass $M$ was not restricted by any approximation. Energy expressions in the small mass (large radius) limit were also presented. For surfaces close to the outer horizon of the Kerr black hole, the quasilocal energy was explicitly calculated under the assumption that $|a| \ll r_{+}$(or equivalently, $|a| \ll M)$. Under these approximations, the curvature of the surfaces involved was positive everywhere and the embedding in $E^{3}$ could be done explicitly. In particular, the quasilocal energy within constant radius surfaces and spherical surfaces is a monotonically decreasing function of the radius. The energy within the ergosurface is larger than $M$, and the energy at the horizon equals twice the irreducible mass of the black hole. These results constitute a natural extension to stationary black holes of previously known results concerning quasilocal energy for static black holes.

Short of an exact evaluation of (1.1), it is perhaps not unreasonable to look for a quasilocal energy expression (say, for $r=r_{0}$ surfaces) that satisfies the four conditions: (1) $E \rightarrow M$ as $r \rightarrow \infty,(2) E \rightarrow 2 M_{i}$ as $r \rightarrow r_{+},(3) E \rightarrow r_{0}-r_{0}\left(1-2 M / r_{0}\right)^{1 / 2}$ as $a \rightarrow 0$, and (4) $E \rightarrow$ Eqn.(3.18) in the limit $|a| \ll r_{0}$. Whereas it is easy to find expressions that fulfill conditions (1)-(3), it is difficult to satisfy (4). In particular, relations of the form $r_{0}-r_{0}\left(1-r_{+} / r_{0}\right)^{1 / 2}$ or $r_{0}-r_{0}\left(1-2 M_{i} / r_{0}\right)^{1 / 2}$ do not satisfy the above criteria. In any case, the desired expression for the quasilocal energy of the Kerr space $\Sigma$ has to reflect the peculiarities of the embedding of boundary surfaces in $E^{3}$ in the fast rotation regime. We hope to return to this issue elsewhere. 


\section{ACKNOWLEDGMENTS}

It is a pleasure to thank Werner Israel for his encouragement and for his critical reading of the manuscript. Research support was received from the Natural Science and Engineering Research Council of Canada through a Canada International Fellowship. 


\section{REFERENCES}

[1] J. D. Brown and J. W. York, Jr., Phys. Rev. D47, 1407 (1993).

[2] R. P. Kerr, Phys. Rev. Lett., 11, 237 (1963).

[3] J. W. York, Jr., Phys. Rev. D33, 2092 (1986); B. F. Whiting and J. W. York, Jr., Phys. Rev. Lett., 61, 1336 (1988).

[4] J. D. Brown, E. A. Martinez, and J. W. York, Jr., Phys. Rev. Lett., 66, 2281 (1991); in Nonlinear Problems in Relativity and Cosmology, edited by J. R. Buchler, S. L. Detweiler, and J.R. Ipser (New York Academy of Sciences, New York, 1991).

[5] J. Katz, D. Lynden-Bell and W. Israel, Class. Quantum Grav. 5, 971 (1988).

[6] S. A. Hayward, Phys. Rev. D49, 831 (1994).

[7] G. W. Gibbons and S. W. Hawking, Phys. Rev. D15 , 2752 (1977).

[8] J. D. Brown and J. W. York, Jr., Phys. Rev. D47, 1420 (1993).

[9] R. Arnowitt, S. Deser, and C. W. Misner, in Gravitation: An Introduction to Current Research, edited by L. Witten (Wiley, New York, 1962).

[10] R. H. Boyer and R. W. Lindquist, J. Math. Phys. 8, 265 (1967).

[11] C. W. Misner, K. S. Thorne and J. A. Wheeler, Gravitation, W. H. Freeman and Sons, San Francisco, 1973.

[12] L. Smarr, Phys. Rev. D7, 289 (1973).

[13] D. Christodoulou and R. Ruffini, Phys. Rev. D4, 3552 (1971). 


\section{FIGURE CAPTIONS}

Figure 1: Embedding diagram (in three-dimensional Euclidean space $E^{3}$ ) of a twodimensional surface whose intrinsic geometry coincides with the intrinsic geometry of a $r=r_{0}$ surface in the Kerr space $\Sigma$. The figure corresponds to particular values of $M$ and $a$ that satisfy the condition $a^{2} \ll r_{0}^{2}$. For fixed $a^{2}$, the surface becomes more oblate as $M$ increases.

Figure 2: The quasilocal energy as a function of the coordinate radius $r_{0}$ of ${ }^{2} B$ in the case $|a| \ll r_{+}$.

Figure 3: The quasilocal energy as a function of the curvature radius $R$ of a round spherical surface for the case $M=1,|a|=.2$. The energy approaches $M$ at spatial infinity. 
This figure "fig1-1.png" is available in "png" format from: http://arxiv.org/ps/gr-qc/9405033v2 
This figure "fig1-2.png" is available in "png" format from: http://arxiv.org/ps/gr-qc/9405033v2 
This figure "fig1-3.png" is available in "png" format from: http://arxiv.org/ps/gr-qc/9405033v2 